\title{
Les Prépositions entre et parmi dans un corpus de textes littéraires contemporains
}

\section{The Examination of the Prepositions entre and parmi in a Corpus of Contemporary Literary Texts}

Iva DeDKovÁ [iva.dedkova@osu.cz]

Ostravská univerzita v Ostravě, République tchèque

\section{RÉSUMÉ}

Larticle est consacré à l'emploi des prépositions entre et parmi dans un corpus composé de sept textes littéraires provenant du français contemporain, qui ont été publiés en 2013 et sont accessibles dans la base textuelle Frantext. Il analyse leurs occurrences, leurs principales propriétés morphosyntaxiques et leurs différents sens et valeurs. Il présente également les résultats d'une enquête linguistique qui a été effectuée dans le milieu universitaire français en décembre 2016 et en avril 2017. Les réponses de 155 sujets, étant pour la plupart des étudiants universitaires, nous ont permis de faire quelques observations intéressantes en ce qui concerne leurs emplois et leurs évocations (à titre d’exemple, la préposition entre peut appeler la locution au milieu (de), la préposition parmi un ensemble dont fait partie quelque chose ou quelqu'un).

\section{MoTS-CLÉS}

préposition entre; préposition parmi; conjonction et ; corpus ; tâche expérimentale

\begin{abstract}
The paper focuses on the use of the prepositions entre [between] and parmi [among] in a corpus consisting of seven literary texts from contemporary French, which were published in 2013 and are accessible through the Frantext database. It analyses their occurrences, frequency of use, morphosyntactic features, differing meanings and function (among which the spatial use of parmi). It also presents the results of an experimental task composed of four sub-tasks and conducted in the French academic milieu in December 2016 and in April 2017. The data gathered from 155 subjects, mostly university students, allowed us to make several interesting observations on their use and linguistic associations (for example, entre can evoke the expression in the middle (of) and parmi the notion of a whole that something or somebody is a part of).
\end{abstract}

\section{KEYWORDS}

preposition entre [between]; preposition parmi [among]; conjunction et [and]; corpus; experimental task 


\section{Introduction}

Le présent article se focalise sur l'emploi des prépositions entre et parmi dans un corpus de textes littéraires provenant du français contemporain et s'inscrit, comme recherche partielle, dans nos études prépositionnelles. De nombreux travaux, en sémantique cognitive, sur les prépositions ont été menés, néanmoins peu d’entre eux se sont focalisés sur ces dernières. À cette occasion, il convient de mentionner celui de Song Nim Kwon-Pak (2006) qui s'intéresse aux contrastes distributionnels et sémantiques des constructions partitives comportant entre et parmi, celui de Philippe Gréa (2015) qui porte sur les perspectives selon lesquelles les prépositions entre et parmi conçoivent la pluralité, ou ceux de Emilia Hilgert $(2011$; 2012) qui s'interroge sur l'interprétation spatiale des prépositions ensemblistes parmi, entre et d'entre, c'est-à-dire celles qui ont pour régime un syntagme nominal pluriel et renvoient à un ensemble.

Le présent article vise à analyser la distribution, les propriétés morphosyntaxiques et les valeurs des deux prépositions concernées dans un corpus disponible grâce à la base textuelle Frantext. Notre corpus est composé de sept œuvres publiées en 2013, parmi lesquelles une biographie, un roman, un journal, deux récits et deux essais ${ }^{1}$. Plusieurs genres de textes de la littérature française contemporaine font l'objet d'une étude conjointe, les critères les plus importants étant leur caractère littéraire ainsi que leur date de publication : cela nous a permis de recueillir un échantillon riche d’emplois actuels et variés de ces deux prépositions. Les exemples étudiés dans cet article seront ci-après ainsi référencés : (E ou $\mathrm{P}: \mathrm{n}^{\circ}$ de l'exemple dans notre corpus). Une tâche expérimentale concernant la problématique traitée a été réalisée dans le milieu universitaire français en décembre 2016 et en avril 2017 et ses résultats seront détaillés dans la dernière section.

\section{Principales propriétés morphosyntaxiques des prépositions entre et parmi en terme de relation}

Les termes entre et parmi $i^{2}$ font partie des principales prépositions françaises. Nous avons observé la fréquence de leurs emplois dans notre corpus de travail, constitué de sept textes littéraires sortis en 2013. Ce corpus contient 273263 mots. Le terme entre y apparait 173 fois, dont 18 fois en tant que forme verbale entre (jentre: 9 occurrences, il/elle/on entre : 9 occurrences) et 1 fois en qualité

1 Voici la liste des œuvres : Artières, P. (2013) : Vie et mort de Paul Gény; Dupuy, A. (2013) : Journal d’une lycéenne sous l'Occupation: Toulouse, 1943-1945 ; Fallet, R. (2013) : Le Vélo ; Garat, A.-M. (2013) : La Première fois ; Garat, A.-M. (2013) : Tranquille; Picquet, P. (2013) : Sans illustration; Simonet, M. - Olivès, F. (2013) : Marc Beltra : roman autour d'une disparition.

2 Rappelons leurs origines, comme les décrit Rey (dir. 1992 \& 2010 : 700 \& 1433). La préposition entre est, à la fin du $\mathrm{X}^{\mathrm{e}}$ siècle, héritée du latin inter (formé de in « dans » et de -ter), signifiant "à l'intérieur de deux » et utilisé au sens spatial et temporel. Parmi, préposition composite, est la lexicalisation, au XII ${ }^{\mathrm{e}}$ siècle, de per mei (fin $\mathrm{X}^{\mathrm{e}}$ siècle), par mi (1080), signifiant «par le milieu, au milieu ». Autrefois, parmi pouvait régir un nom singulier (par ex. parmi la campagne, parmi ce grand amour [Corneille] ; emploi aujourd'hui qualifié de vieilli ou littéraire) et avait, d'après Grevisse - Goosse (2008 : 1371), deux synonymes : emmi et entremi. Notons quau cours de leur évolution, les emplois des deux prépositions ont été grammaticalisés, c.-à-d. que leurs usages ont été fixés, mais les locuteurs semblent sêtre appropriés ces prépositions de manière plus personnelle, ce qui pourrait également contribuer à expliquer certains usages décalés en fonction des autres sens de entre et parmi. 
de la préposition espagnole entre ${ }^{3} .9$ fois, entre fait partie de deux mots composés : entre-temps (7 occurrences) et entre-deux-guerres (2 occurrences). Entre est donc employé prépositionnellement 145 fois : seul, c.-à-d. comme préposition simple (131 occurrences), ou précédé de la préposition de (14 occurrences). Sur l’ensemble des occurrences des prépositions simples et leurs expressions homonymiques, dont le nombre total sélève à 25243 , la forme entre occupe le $15^{\mathrm{e}}$ rang et présente ainsi une fréquence dans l'usage de 0,69\%. En revanche, la préposition parmi na que 34 occurrences : elle occupe la $23^{\mathrm{e}}$ place et présente une fréquence dans l'usage de $0,13 \%$. Notons que, dans la liste de fréquence élaborée par Vaguer $(2008: 23)$, entre occupe la $12^{\mathrm{e}}$ place et parmi la $23^{\mathrm{e}}$, dans celle du Français fondamental, entre est classé au $8^{\mathrm{e}}$ rang et parmi à nouveau au $23^{\mathrm{e}}$, ce qui en fait une préposition de faible fréquence.

Ci-dessous, la liste de fréquence des prépositions simples les plus typiques du français, y compris leurs expressions homonymiques, qui sont attestées dans notre corpus :

\begin{tabular}{|c|c|c|}
\hline $\mathrm{N}^{\circ}$ & Prép. & \\
\hline 1 & de & 8989 \\
\hline 2 & à & 4526 \\
\hline 3 & en & 2762 \\
\hline 4 & dans & 1919 \\
\hline 5 & pour & 1525 \\
\hline 6 & sur & 1123 \\
\hline 7 & avec & 1038 \\
\hline 8 & par & 893 \\
\hline 9 & sans & 412 \\
\hline
\end{tabular}

\begin{tabular}{|c|c|c|}
\hline $\mathrm{N}^{\circ}$ & Prép. & \\
\hline 10 & après & 357 \\
\hline 11 & sous & 207 \\
\hline 12 & depuis & 200 \\
\hline 13 & avant & 180 \\
\hline 14 & vers & 177 \\
\hline 15 & entre & 173 \\
\hline 16 & pendant & 169 \\
\hline 17 & devant & 159 \\
\hline 18 & chez & 134 \\
\hline
\end{tabular}

\begin{tabular}{|c|c|c|}
\hline $\mathrm{N}^{\circ}$ & Prép. & \\
\hline 19 & dès & 65 \\
\hline $20-21$ & contre & 58 \\
\hline $20-21$ & derrière & 58 \\
\hline 22 & selon & 49 \\
\hline 23 & parmi & 34 \\
\hline 24 & hors & 21 \\
\hline 25 & jusque & 9 \\
\hline 26 & envers & 6 \\
\hline Total & 26 prép & 25243 \\
\hline
\end{tabular}

Au sujet de ses propriétés combinatoires, la préposition entre implique un syntagme nominal (nom ou pronom) et des régimes pluriels, qui peuvent être des coordinations de singuliers. Elle peut concerner deux ou plusieurs éléments (elle n'est donc pas seulement utilisable lorsqu'elle est suivie de deux entités), par exemple : $^{4}$

entre (dét) $\mathrm{N}_{\mathrm{pl}}$ : Travailler en archive, c'est sans cesse être entre ces deux massifs et se tenir en équilibre (E: 27).

entre (dét) $\mathrm{N}_{\mathrm{sg} / \mathrm{pl}}$ et (dét) $\mathrm{N}_{\mathrm{sg} / \mathrm{pl}}$ : Dans ces années-là, 1978-1979, il n'y avait pas d'accords d'exequatur entre la France et l'Algérie (E : 127).

entre (dét) $\mathrm{N}_{\mathrm{sg} / \mathrm{p} \mathrm{p}^{\prime}}$ (dét) $\mathrm{N}_{\mathrm{sg} / \mathrm{pl}}$, (dét) $\mathrm{N}_{\mathrm{sg} / \mathrm{pl}}$ et (dét) $\mathrm{N}_{\mathrm{sg} / \mathrm{pl}}$ : Bataille avec les feuilles de fusain qui ornent la nappe entre Janine, Lopez, Paulette et moi (E : 76).

entre (dét) $\mathrm{N}_{\mathrm{sg} / \mathrm{pl}}$, (dét) $\mathrm{N}_{\mathrm{sg} / \mathrm{p} p}$ (dét) $\mathrm{N}_{\mathrm{sg} / \mathrm{pl}}$, (dét) $\mathrm{N}_{\mathrm{sg} / \mathrm{pl}}$ et (dét) $\mathrm{N}_{\mathrm{sg} / \mathrm{pl}}:$ [...], mes vacances étant inégalement partagées entre le cycle, la pétanque, la pêche, la lettre d'amour - les écureuils pensent à l'hiver - et les tournées rurales d'apéritifs à la terrasse de mon Q.G. (E : 90).

3 Tu sais quéen écoutant la radio j’ai entendu: "Sufre mamón, devuélveme a mi chica, o te retorcerás entre polvos pica » [...] (E : 173). Notons que ces paroles sont tirées d'une chanson du groupe Hombres G.

4 Aucun exemple attesté dans notre corpus pour la construction entre (dét) $N_{s g / p l}$ (dét) $N_{s g / p l}$ et (dét) $N_{s g / p l}$. 
Dans les phrases de notre corpus, entre introduit :

i. un nom pluriel, avec ou sans déterminant : entre femmes, entre les mains, entre quatre murs, entre ces trois pays, entre plusieurs enfants,

ii. un pronom pluriel : entre nous, entre eux, entre ceux, entre autres, entre nous deux,

iii. deux ou plusieurs syntagmes nominaux reliés par la conjonction et : entre le personnel et les patients, entre le balcon et nous, entre 1961 et 1969,

iv. une proposition infinitive, comparable sémantiquement à un syntagme nominal (c.-à-d. le fait de ... $)^{5}$ : (J'hésitais) entre mexexcuser (E : 165).

La phrase J'hésitais entre m'excuser est agrammaticale par l'utilisation d'une préposition inappropriée. L'auteur aurait voulu dire : j’hésitais à m'excuser, quand je mexcusais, je me demandais si javais raison de le faire. Il est possible d'envisager que la construction de son propos était la suivante : j'hésitais entre lui présenter mes excuses ou pas.

Dans l'exemple suivant, entre donne l'impression d'introduire un seul nom singulier. En effet, syntaxiquement, il s'agit d'une coordination de deux syntagmes nominaux composés d'un nom et d'un syntagme prépositionnel complément de nom ; le nom « le bruit » dans le deuxième syntagme nominal est élidé :

(2.1) On savait faire la différence entre le bruit d’une grenade et de la mitraille (E:61).

La préposition entre peut être précédée de la préposition de. La forme d'entre apparaît 14 fois dans notre corpus : 13 fois, elle est suivie d'un pronom pluriel (il s'agit de la construction partitive : quantificateur $+\mathrm{d}^{\prime}+$ entre + Pro $_{\mathrm{pl}}$ ), et une fois, d'un nom pluriel (la construction : $\mathrm{V}+\mathrm{d}^{\prime}+$ entre $\left.+\mathrm{N}_{\mathrm{pl}}\right)$ :

i. (peu / nombre / la majorité / un / l'un / quelques-uns) d'entre eux (6 occurrences),

ii. (chacun / bien peu / l'un / l'une) d'entre nous (4 occurrences),

iii. (nombre / l'une) d'entre elles ( 2 occurrences),

iv. (certains) d'entre vous (1 occurrence),

v. d'entre les mélèzes (1 occurrence).

Rappelons que d'entre s'emploie après des expressions quantitatives, obligatoirement si le quantificateur est au pluriel, ${ }^{6}$ facultativement si le quantificateur est au singulier, ${ }^{7}$ par exemple :

5 Voici un autre exemple, présenté par Franckel et Paillard (2007 : 38), qui le classent dans la catégorie où « Y correspond à deux $\mathrm{N}$ ou deux Pronoms reliés par et » : Hésiter entre aller à la Fac et passer chez Jacques.

6 Notons que, par exemple, "peu » est un adverbe, donc il est difficile de dire qu'il est au pluriel : Peu d'entre eux sont arrivés à vélo $(\mathrm{E}: 95) . \rightarrow{ }^{*}$ peu d’eux.

7 Voici un autre exemple tiré du corpus : À la suite de quelques paroles un peu vives échangées entre plusieurs enfants de dix ans à douze ans dans le quartier des Pénitents blancs, l’un d’eux, le jeune Bisceto Auguste, âgé de douze ans, a pris le fusil de chasse de son père, [...] (E: 35). $\rightarrow$ l'un d'entre eux. 
(2.2) Je rêve que certains d'entre vous (un sur cent? un sur mille?) aient envie, en refermant ce livre, d'apporter ce qu'ils savent, pour aider Françoise (E: 163). $\rightarrow{ }^{*}$ certains de vous

(2.3) En couture, l'une d'entre nous était chargée de lire (E : 50). $\rightarrow$ l'une de nous

Une seule fois, il s'emploie au sens spatial pour marquer une idée de passage :

(2.4) En approchant, d’entre les mélèzes, j’ai vu un filet de fumée sortir du toit, tout droit, tranquille; j’ai su qu'il était toujours là (E : 119). ${ }^{8}$

La préposition parmi, dans notre corpus, régit un syntagme nominal pluriel renvoyant à plus de deux éléments :

i. un nom pluriel, avec déterminant (19 occurrences) : parmi les normaliennes, parmi toutes les erreurs, parmi ces milliards de vivants, parmi les débris de son appareil, ${ }^{9}$

ii. un pronom ${ }^{10}$ pluriel (14 occurrences) : parmi eux (7 attestations), parmi lesquels/lesquelles (4 attestations), parmi (beaucoup) d'autres (2 attestations), parmi ceux-ci (1 attestation),

iii. un nom collectif, suivi d'un complément pluriel (1 occurrence) : parmi un panel de photographies.

Contrairement à entre, parmi peut introduire les noms collectifs. ${ }^{11}$ Mais cet usage n'est attesté qu'une seule fois dans notre corpus. Dans l'exemple suivant, le nom collectif " panel » sert à définir un nombre de photographies, car employé comme déterminant nominal de quantité, et l'accord se fait au singulier :

(2.5) Janina confirme que Marc était présent à Nova Zelanda en décembre 2003 (elle a immédiatement reconnu son visage parmi un panel de photographies qui était posé sur une table) (P : 33).

Parmi introduit principalement des entités multiples. Voici un seul cas limite tiré de notre corpus dans lequel parmi ne régit que deux ou trois participants :

(2.6) Il y avait deux ou trois personnes, parmi lesquelles madame A..., venue de mon lointain village $(\mathrm{P}: 23)$.

À ce propos, Grevisse - Goosse (2008 : 1371) affirment que : «S’il y a une indication numérique, elle n’est pas inférieure à trois. Ex. tout à fait exceptionnel : Ta démission produit le plus mauvais effet parmi ces deux messieurs (Valéry, Corresp., cit. Trésor) ».

8 Voici le test d'application avec les termes d'où et en pour prouver l'utilisation spatiale de la forme d'entre : D'où approchais-je et d’où ai-je vu un filet de fumée ? Il y avait des mélèzes : j’en approchais et j’en ai vu (en = d’entre les mélèzes).

9 Les pluralia tantum ont tendance à suivre parmi (voir Gréa $2015: 20$ ).

10 Parmi n'admet pas le pronom relatif qui.

11 Voici un autre exemple : Nîmes : intoxication massive parmi le personnel de l'hôpital (Le Parisien 2017). C'est-à-dire parmi les membres du personnel. 
La structure avec deux syntagmes nominaux coordonnés, parmi les $S N$ et les $S N$, telle que se promener parmi les arbres et les feuilles mortes, n'apparait pas dans notre corpus.

Enfin, la combinatoire des prépositions entre et parmi se limite à un complément de type syntagme nominal, ce qui les rapprocherait, sur le plan syntaxique, des prépositions chez, envers et sous. D’après Grevisse - Goosse (2008 : 1328-9), l'emploi absolu de entre appartient à l'usage familier et celui de parmi est rarissime. Aucun des deux ne fait partie de notre corpus.

\section{Sens et différents emplois des prépositions entre et parmi}

Les deux prépositions, entre et parmi, pourraient être classées dans la catégorie des prépositions pleines, aussi dites colores ou lexicales (voir p. ex. Cadiot 1997 : 36, ou Kwon-Pak 2006 : 652), d’après la limpidité de leurs sens.

Selon les 155 sujets qui ont participé à notre tâche et dont les résultats sont détaillés dans la quatrième section, la préposition entre évoque, en premier lieu, la locution au milieu (de) (55 réponses), et la préposition parmi fait référence à un ensemble dont fait partie quelque chose ou quelqu'un (31 réponses).

Les deux prépositions expriment l'inclusion, mais chacune englobe différentes nuances de sens. La préposition entre introduit un ou plusieurs ensembles d'éléments qui sont liés par un rapport spécifique. Parmi, au contraire, n'est pas marqué par ce trait d’interconnexion des constituants formant la totalité, ses constituants particuliers sont plus au moins autonomes (à ce propos, voir p. ex. Gréa 2015, ou Kwon-Pak 2006). Autrement dit, entre crée des regroupements suivant des critères homogènes et basés sur la dépendance fonctionnelle des éléments les uns avec les autres, ce qui n'est pas le cas de parmi qui opère des regroupements d'après des critères hétérogènes et rejette des rapports de liaison fonctionnels interélémentaires. C'est pourquoi, dans les deux exemples suivants, seule la préposition entre est possible, parmi étant exclu, car il s'agit des deux mains d'une personne dans (3.1) et des quatre murs d'une même pièce dans (3.2) :

(3.1) Il tient ma main gauche entre ses deux mains et je ne le sentais pas (E : 130).

(3.2) Entre les quatre murs du labo photo, elle jaillit avec la primeur d'alors, [...] (E : 116).

D'après Martinet (dir., 1979 : 185), entre accentue l'entourage, alors que parmi souligne l'objet en cause par rapport à cet entourage. Selon Gréa (2015 : 16), la cible de entre est localisée par rapport aux éléments du site, la cible de parmi, par rapport au tout. ${ }^{12}$

La préposition entre connaît des emplois spatiaux, temporels et notionnels, alors que parmi ne s'applique qu'aux domaines spatial et temporel.

12 La nuit (= la cible) tombe entre Le Garric et Carmaux (= le site) (E : 68). 


\section{1 À propos de la préposition entre}

Dans un premier temps, la préposition entre se voit employer au sens spatial, pour désigner un espace intermédiaire délimité par deux ou plusieurs éléments ordonnés (c’est-à-dire que la cible se trouve dans un intervalle de localisation entre deux ou plusieurs ensembles) ${ }^{13}$. Il peut s'agir d'un espace unique, comme dans (3.3) ou (3.4), ou d'une succession d'espaces, comme dans (3.5) ou (3.6). La valeur spatiale apparaît fréquemment dans notre corpus et peut être prouvée par l'application d'un test basé sur les reformulations avec les termes où et $y^{14}$ :

(3.3) À peine débouchait-il sur la place qu'il était happé par la foule qui s’y pressait; il avait beau faire, entre les calèches, les grappes de touristes et les vendeurs de babioles et de roses, il se frayait difficilement un passage ( $\mathrm{E}: 40)$.

$\rightarrow$ Test : Où avait-il beau faire et se frayait-il difficilement un passage ? Il y avait beau faire et s'y frayait difficilement un passage ( $\mathrm{y}=$ entre les calèches, les grappes de touristes et les vendeurs de babioles et de roses).

(3.4) Chez Élia, nous apprenons que le débarquement sest enfin produit entre Dunkerque et Cherbourg et ce nest pas un bobard c'est la radio qui l’a annoncé (E : 54).

$\rightarrow$ Test : Où le débarquement s'est-il enfin produit ? Le débarquement s'y est enfin produit ( $\mathrm{y}=$ entre Dunkerque et Cherbourg).

(3.5) [...] d'amères poraganes [c.-à-d. poireaux sauvages] poussés entre les rangs de vigne [...] (E : 103). $\rightarrow$ Test : Où poussent d’amères poraganes ? Ils y poussent ${ }^{15}(\mathrm{y}=$ entre les rangs de vigne).

(3.6) Avec lui, nous avons rouvert les volets, [...], passé la tête-de-loup entre les poutres pour chasser les toiles d'araignées, longs voiles de veuve flottant au courant d'air ; [...] (E : 106).

$\rightarrow$ Test : Où avons-nous passé la tête-de-loup ? Nous y avons passé la tête-de-loup ( $\mathrm{y}=$ entre les poutres).

Entre, de même que parmi, s'emploie dans l'expression spatiale statique ainsi que cinétique (procès). Celle-ci n'est pas directement exprimée par la préposition elle-même, mais essentiellement par le verbe. Les exemples (3.3), (3.5) et (3.6) ci-dessus illustrent l'utilisation cinétique (procès), les exemples (3.4) et (3.7)-(3.9) l'utilisation statique :

(3.7) Je me retrouve dans la salle de chimie, au premier rang, entre Josette et Antoinette (E : 43). ${ }^{16}$

(3.8) Je le voyais comme un paradis, car ma chambre était coincée entre deux pièces (E : 59).

13 «Entre crée l'idée de frontières d’un ensemble fermé [...] », précise Hilgert (2011:3).

14 Le terme $y$ peut reprendre également des compléments non spatiaux : elle y pense, à son avenir ; elle y est hostile, à cette décision.

15 D'un point de vue sémantique, il s'agit d'un procès.

16 Avec deux syntagmes nominaux, entre, à la différence de parmi, accepte des marqueurs adverbiaux (voir Hilgert $2012: 6)$ : ... entre Josette, à ma gauche, et Antoinette, à ma droite. 
(3.9) Pour y accéder, il fallait qu'il embarque sur le rio Javari, fleuve qui constitue une frontière naturelle entre le Pérou et le Brésil (E : 139).

D’après Hilgert (2012 : 14), " entre exprime l'ordonnancement des éléments de l'ensemble et la saturation de tous les espaces créés par des oppositions duelles démultipliées ». Voici deux exemples illustratifs. Dans (3.10), l'effet d'une opposition duelle démultipliée est créé par la répétition de la préposition entre. Dans (3.11), il n'est pas certain qu'il s'agisse d'une ou de plusieurs rangées de haies vives. Dans le cas où il y aurait plusieurs rangées de haies vives, celles-ci pourraient être continues ou discontinues. Voir aussi l'exemple (3.3), dans lequel entre introduit trois ensembles (il avait beau faire entre les calèches, les grappes de touristes et les vendeurs de babioles et de roses). La préposition entre peut impliquer l'idée d'une " opposition duelle démultipliée ", son usage, cependant, ne semblerait pas aussi strict.

(3.10) Arrivée à Saint-Sulpice : arrêt indéterminé (la voie a sauté entre Saint-Sulpice et Rabastens, et entre Rabastens et Lisle-sur-Tarn.) (E: 52-53).

(3.11) Nous prenons un petit chemin qui monte et descend entre les haies vives (E : 69).

Dans un deuxième temps, entre est utilisé au sens temporel pour désigner un intervalle de temps délimité par deux moments, c'est-à-dire qu'il situe un événement dans un intervalle temporel (voir Franckel-Paillard 2007 : 54). Cet emploi est assez fréquent dans notre corpus et les deux structures typiques sont entre (dét) $N_{s g}$ et (dét) $N_{s g}$ et entre dét $N_{p l}$ :

(3.12) Entre le poulet et le dessert, Christo chante, puis Régine ("Quand allons-nous nous marier?»), Janine, Josette, Bruzy (« La Guitare à Chiquita ») (E: 75).

(3.13) Parce que entre la libération de Toulouse et mai 1945, la guerre avait continué (E : 84).

(3.14) Combien de secondes entre le moment où le métro ralentit et l'ouverture des portes? (E : 134).

(3.15) Entre Noël et le jour de l'an, Françoise s'est rendue au commissariat du XI arrondissement de Paris pour faire part de la disparition de son fils (E : 140).

(3.16) La seconde mission de la brigade criminelle a eu lieu entre le 11 et le 23 novembre 2006 (E: 158).

(3.17) Entre 2004 et 2006, on prenait le métro côte à côte : je prenais des notes; il prenait des photos (E : 167).

(3.18) Je le [= le vélo] revois entre deux métros, deux rendez-vous, et je revois les routes qui le connaissent bien, et je souffre sur elles, et j’ai faim de vélo, [...] (E : 101). 
(3.19) Entre deux séances de chimio, elle écoutait parfois Marco, en balançant son pied droit, une cigarette à la bouche : "J'adore... » (E : 156).

Comme nous pouvons le voir ci-dessus, l'expression temporelle peut délimiter un intervalle plus ou moins précis, comme entre Noël et le jour de l'an dans (3.15), ou un intervalle plus vague, tel que entre deux métros, deux rendez-vous dans (3.18).

Dans l'exemple suivant, il pourrait s'agir d'une succession d'intervalles temporels :

(3.20) Entre les alertes, on déjeunait ( $\mathrm{E}: 60)$.

À propos de la fonction temporelle de entre, Martinet (dir. 1979 : 194-195) affirme qu' : " Elle est normalement une réponse à quand?, mais également à combien de temps ? Cependant, il n'y a pas lieu de poser, pour la réponse à ce dernier, une fonction temporelle particulière ; il s'agit en fait d'une extension, à des réalités temporelles, de la fonction spatiale, Cela durera entre quatre et six jours étant du même type que Il est assis entre deux chaises. "

La préposition entre s'utilise aussi pour désigner des valeurs notionnelles, par exemple lapproximation :

(3.21) On dit que ces placard carriers gagnent aujourd'hui entre 2 et 4 livres sterling par heure pour demeurer toute la journée sur une artère commerciale comme Oxford Street (E : 24).

(3.22) "Je mange des guins", c’est une sorte de fruit entre la guigne et la cerise (E : 57).

Les placard carriers, dans (3.21), gagnent de 2 à 4 livres sterling. Dans (3.22), il s'agit d'une sorte de fruit qui ressemble en partie à la guigne et en partie à la cerise.

Entre indique des relations réciproques diverses (amitié ou hostilité, similitude ou différence, opposition, etc. $)^{17}$. Dans les structures comportant le coordonnant et, entre oppose deux (éventuellement plusieurs) ensembles, soulignant un trait particulier, comme la nationalité, le genre, le statut social ou la profession - Français versus Anglais, filles versus garçons, patrons versus ouvriers, avocat versus écrivain :

(3.23) On y voit le combat entre Français et Anglais et ces derniers nont pas le beau rôle (E : 49).

(3.24) Les relations entre filles et garçons se sont émancipées (E : 81).

(3.25) En 1936, la libération s'est opérée d'un point de vue social, entre patrons et ouvriers, entre l'État et ses fonctionnaires (E : 82-83).

(3.26) J'aborderai dans ce livre la "schizophrénie » entre mon oil d'avocat et mon cil d'écrivain (qui nont bien sûr pas la même vision) (E : 148).

17 «[... entre est de façon exclusive marquée par le trait de réciprocité » (Kwon-Pak 2006 : 656). 
Nombre de ces exemples peuvent être paraphrasés à l'aide de la préposition avec, qui désigne le comitatif ou la coprésence (avec est plus actif, entre plus passif), ou de la conjonction de coordination et.

(3.27) Mais aussi quand le contact est établi, quels rapports charmants sétablissent entre l'aumônier et ces grands enfants! (E : 16).

$\rightarrow$ Laumônier commence à avoir un rapport charmant avec ces grands enfants.

(3.28) Il y avait une harmonie entre les rideaux de la fenêtre et la couleur des meubles laqués blancs (E: 58).

$\rightarrow$ Les rideaux de la fenêtre s'accordaient bien avec la couleur des meubles laqués blancs.

(3.29) Bataille avec les feuilles de fusain qui ornent la nappe entre Janine, Lopez, Paulette et moi $(\mathrm{E}: 76) .^{18}$

$\rightarrow$ Bataille avec les feuilles de fusain qui ornent la nappe, avec Janine, Lopez, Paulette et moi.

(3.30) Nous jouions ensemble, entre lycéennes et normaliennes (E : 51).

$\rightarrow$ Les lycéennes jouaient avec les normaliennes. / Les lycéennes et les normaliennes jouaient ensemble.

(3.31) Dispute entre la bouchère et Mme Grave (E : 63).

$\rightarrow$ La bouchère se dispute avec Mme Grave. ${ }^{19}$ / La bouchère et Mme Grave se disputent.

(3.23a) On y voit le combat entre Français et Anglais et ces derniers nont pas le beau rôle (E : 49). $\rightarrow$ Les Français et les Anglais combattent l'un contre l'autre.

Voici trois autres paraphrases possibles :

(3.32) À Ricardie, à Saint-Agne, cétait la compétition entre les écoles pour obtenir le prix Fabre décerné aux meilleures élèves (E : 46).

$\rightarrow$ Les écoles étaient en compétition pour obtenir le prix Fabre.

(3.33) Il y a un petit conflit générationnel entre les deux profs de gym (E: 47).

$\rightarrow$ Les deux profs de gym ont un petit conflit générationnel.

(3.29a) Bataille avec les feuilles de fusain qui ornent la nappe entre Janine, Lopez, Paulette et moi (E : 76). $\rightarrow$ Nous nous battons ensemble, avec les feuilles de fusain qui ornent la nappe.

18 Il faut noter que le sens de cette phrase pourrait être ambigu. S’agit-il d'une bataille entre les personnages, ou les feuilles de fusain seraient-elles posées entre les personnages ? Le premier sens serait clair si on ajoutait deux virgules : Bataille, avec les feuilles de fusain qui ornent la nappe, entre Janine, Lopez, Paulette et moi.

19 On peut également changer de focus discursif, par ex. : Mme Grave se dispute avec la bouchère. Les normaliennes jouaient avec les lycéennes. 
Dans les exemples suivants, entre marque un regroupement de personnes, en excluant toute autre, c.-à-d. une appartenance à un ensemble homogène :

(3.34) Nous partions entre femmes faire un peu de marché noir (E : 70).

(3.35) Nous organisions des « courses », entre copains, [...] (E : 94).

Entre aide à exprimer l'appartenance à un ensemble, homogène dans le premier cas (c.-à-d. coureurs) ou plutôt hétérogène dans le second (c.-à-d. plusieurs accusations), entre autres voulant dire « en particulier, notamment» :

(3.36) J'ai demandé à des coureurs, à Jacques Anquetil entre autres, s'ils avaient débusqué dans leur carrière, au sein des pelotons, un coursier équivoque, grimpeur au patchouli ou sprinter languissant du cuissard ( $\mathrm{E}: 88)$.

(3.37) Je soupçonne le bougre de confondre chevesne et truite, mouches sèches et noyées, de se gausser des tailles réglementaires des poissons nobles, l'accuse entre autres d'emploi d'appâts illicites, mais, comme disait Feydeau, "ça n'empêche pas l'estime " (E: 91).

Dans l'exemple suivant, entre codésigne un état, sens rarissime dans notre corpus - « être entre deux eaux » signifiant ici " être entre deux réels ", en évoquant l'instant où le patient passe du sommeil à létat de veille :

(3.38) Je suis « entre deux eaux», flottant encore dans les brumes de l’anesthésie (E : 133).

Le syntagme l’épreuve entre toutes étrange, ci-dessous, signifie «l'épreuve étrange par excellence»:

(3.39) [...] et l'on oublie combien nous refaisons peu ou prou leurs gestes d'opticiens et de chimistes afin d'obtenir peut-être l'épreuve entre toutes étrange par laquelle se présente une deuxième fois la vue des lieux, des objets et des êtres absents, éloignés dans le temps, morts ou disparus, [...] (E : 113).

Enfin, le complément circonstanciel avec la préposition entre peut suggérer des informations variées, par exemple une notion temporelle et une notion spatiale, comme dans l'exemple (3.40):

(3.40) Nous partons vers 18 h. [...] La nuit tombe entre Le Garric et Carmaux. Changement à Carmaux. Le wagon de $2^{\text {de }}$ est en face de notre portière (E : 68). 
Dans l'exemple (3.41), le syntagme prépositionnel comportant entre pourrait évoquer ou le lieu, ou la manière :

(3.41) À Madrid, il n'est plus toléré de déambuler dans les rues entre des panneaux d'affichage publicitaire, c'est-à-dire en «sandwich» $(\mathrm{E}: 22)$.

En ce qui concerne d'entre, dans les deux exemples suivants, celui-ci codésigne la division d'un ensemble, c.-à-d. mes collègues et les mendiantes, en extrayant un sous-ensemble, c.-à-d. nombre d'entre eux et l'une d'entre elles ${ }^{20}$ :

(3.42) Je doute que mes collègues historiens comprennent vraiment ma démarche. Bien que nombre d'entre eux soient des catholiques convaincus, je nai rien dit de ma vie religieuse (E:8).

(3.43) [...] les mendiantes miappellent "padre » et l'une d'entre elles me propose de me tirer la bonne aventure $(\mathrm{E}: 11)$.

\section{2 À propos de la préposition parmi}

La préposition parmi désigne, au sens propre, le lieu : " au milieu de » (voir Grevisse - Goosse 2008 : 1371). D’après Martinet (dir. 1979 : 186), parmi combine les traits d'intériorité et de sélection et suggère des choix. Selon Hilgert (2012: 14), parmi « signifie la dissociation des éléments de l'ensemble localisateur et l'occupation partielle et aléatoire des espaces que ces éléments délimitent entre eux ». Kwon-Pak (2006 : 653) remarque que l'emploi spatial de parmi est souvent considéré comme vieilli ou littéraire.

Dans les exemples suivants, parmi indique un rapport spatial, ce que prouve un test fondé sur les reformulations avec les termes où et $y$, et évoque l'idée de plusieurs éléments disséminés, disposés aléatoirement ${ }^{21}$ :

(3.44) C'est pourquoi il établit sa résidence dans un lieu solitaire, parmi les propriétés foncières que ses parents lui avaient léguées, entre le faubourg de la ville et le coteau qui porta plus tard le nom de Sainte-Croix (P : 6).

$\rightarrow$ Test : Où établit-il sa résidence ? Il y établit sa résidence ( $\mathrm{y}=$ parmi les propriétés foncières).

(3.45) C'est avec une poignante émotion que tous les Italiens ont appris qu’on venait de découvrir, parmi les débris de son appareil, les restes d’un aviateur tombé au cours de la guerre (P : 10).

$\rightarrow$ Test : Où venait-on de découvrir les restes d'un aviateur ? On venait d'y découvrir les restes d'un aviateur ( $\mathrm{y}=$ parmi les débris de son appareil).

20 Voir également Hilgert (2012: 13). Šabršula (1989: 140) parle de la relation de « sélection ».

21 Le contexte de l'exemple suivant veut dire que l'abeille zigzaguait (elle volait quelque part, ici et là) : Dans le jardin, parmi les fruits et les légumes, une abeille volait. La cible, l'abeille, se déplaçait aléatoirement au milieu du site, c.-à-d. de l'ensemble localisateur, parmi les fruits et les légumes. 
(3.46) À l'automne de cette année-là, je me suis résolue à passer une nuit chez ma mère, la dernière me disais-je, dans cette maison où elle a été enfant, quelle a hérité de son père, lui-même la tenant de son père et celui-ci de son propre père qui la bâtit de ses mains en 1873 pour lui et ses fils au lieu-dit Les Calinottes, un peu à lécart du village dans cette campagne du Médoc parmi les vignes (P : 19).

$\rightarrow$ Test : Où a-t-il bâti la maison ? Il y a bâti la maison ( $\mathrm{y}=$ un peu à l'écart du village + dans cette campagne du Médoc + parmi les vignes).

Précisons que le syntagme parmi les propriétés foncières dans (3.44) appelle l'espace et un choix : dans une des propriétés foncières. Le syntagme entre le faubourg de la ville et le coteau veut dire : dans un espace intermédiaire, délimité par deux éléments, le faubourg de la ville et le coteau.

Le syntagme parmi les débris de son appareil dans (3.45) suggère l'espace et un choix isolé. On peut remplacer parmi par les locutions au milieu de et à l'intérieur de, qui conçoivent un site pluriel comme un tout : à l'intérieur / au milieu des débris.

Le syntagme parmi les vignes dans (3.46) exprime l'idée de plusieurs vignes disposées aléatoirement, quelque part au milieu des vignes, alors que le syntagme entre les vignes voudrait dire qu'il y aurait deux ou plusieurs vignes alignées.

Rappelons que la préposition entre peut suggérer un ensemble homogène, alors que la préposition parmi, un ensemble plutôt hétérogène. Dans la phrase je passe entre les voitures, les voitures sont les mêmes, analogues, et elles sont alignées. Dans la phrase je passe parmi les voitures, les voitures sont différenciées, indéfinies, et je zigzague. ${ }^{22}$

Les traits d'intériorité et de sélection sont également visibles dans les emplois notionnels de parmi, qui évoque un ensemble d'êtres ou de choses souvent conçu de façon non exhaustive ou hétérogène. Ses participants ne sont pas fonctionnellement dépendants et présentent une certaine autonomie les uns par rapport aux autres (voir Gréa 2015 : 14). À titre d’exemple, il peut s'agir d'un regroupement démographique (parmi ces filles - voir 3.48) ou professionnel (parmi les intervenants - voir 3.55).

Parmi aide à exprimer que quelqu'un ou quelque chose se trouve au milieu ou au sein d'un ensemble dêtres ou de choses; les reformulations avec les termes où et $y$ sont ici possibles :

(3.47) Ses parents occupaient un rang distingué parmi les familles de Lectoure ; [...] (P : 5).

$\rightarrow$ Test : Où ses parents occupaient-ils un rang distingué ? Ils y occupaient un rang distingué ( $\mathrm{y}=$ parmi les familles de Lectoure).

(3.48) Je me sens horriblement seule parmi ces filles que jai toujours crues dédaigneuses et fières (P : 13).

$\rightarrow$ Test : Où me sens-je horriblement seule ? Je m’y sens horriblement seule ( $\mathrm{y}=$ parmi ces filles).

(3.49) Je pense qu'avec ses manières, elle se sentira très à l'aise parmi les lycéennes ( $\mathrm{P}: 14$ ).

$\rightarrow$ Test : Où se sentira-t-elle très à l'aise ? Elle s'y sentira très à l'aise ( $\mathrm{y}=$ parmi les lycéennes).

22 Cette différence s'efface en tchèque, où il n'y a qu'une préposition, mezi, qui correspond à ces deux prépositions françaises : procházím mezi auty. Voici quelques autres exemples en rapport avec notre corpus : entre les quatre murs du labo photo - mezi čtyřmi stěnami fotolaboratoře, entre Noël et le jour de l’an - mezi Vánocemi a Novým rokem, le combat entre Français et Anglais - boj mezi Francouzy a Angličany, parmi les débris de son appareil - mezi troskami jeho letadla, parmi les élèves du collège - mezi žáky školy. La forme d'entre correspond à la préposition tchèque $z$ : l'une d'entre elles - jedna $z$ nich. 
Parmi marque également l'appartenance à un ensemble, exhaustif (3.50) ou non exhaustif (3.51 et 3.53), homogène (3.50) ou hétérogène (3.52) :

(3.50) Je compte parmi les heures les plus belles de ma vie celles de mes conversations philosophiques avec lui $(\mathrm{P}: 3)$.

(3.51) Et ainsi je fis le tour de plusieurs villes d'Italie parmi lesquelles principalement Venise d'où jembarquai sur un navire bulgare le S.S. Costantin [...] (P :9).

(3.52) Elle leur a dit qu'elle ne mavait jamais eue comme professeur, mais les a assurées que je jouissais, parmi les élèves du collège, d’une «très bonne réputation "! ( $\mathrm{P}: 25)$.

(3.53) Une scène parmi d'autres (P : 28).

Dans notre corpus, parmi aide aussi à sélectionner ou à isoler un élément d'un groupe :

(3.54) Parmi eux, des hommes en sang, aux maillots, aux cuissards, aux mains, aux coudes déchirés (P : 18).

(3.55) Parmi les intervenants, mon ancien chirurgien le docteur J... (P : 24).

(3.56) Il n'y avait pas de milicien parmi eux (P : 16).

Enfin, les syntagmes prépositionnels comportant parmi et un syntagme nominal pluriel, notamment leurs emplois non spatiaux, peuvent être reformulés avec faire partie de (voir également Gréa $2015: 14)$ :

(3.46a) [...] un peu à l'écart du village dans cette campagne du Médoc parmi les vignes (P : 19). $\rightarrow$ Les vignes font partie de cette campagne du Médoc.

(3.49a) Je pense qu'avec ses manières, elle se sentira très à laise parmi les lycéennes (P : 14). $\rightarrow$ Elle fait partie des lycéennes.

(3.50a) Je compte parmi les heures les plus belles de ma vie celles de mes conversations philosophiques avec lui $(\mathrm{P}: 3)$.

$\rightarrow$ Mes conversations philosophiques avec lui font partie des heures les plus belles de ma vie.

(3.51a) Et ainsi je fis le tour de plusieurs villes d'Italie parmi lesquelles principalement Venise d'où jembarquai sur un navire bulgare le S.S. Costantin [...] (P : 9).

$\rightarrow$ Venise fait partie des villes d'Italie dont je fis le tour. 
(3.55a) Parmi les intervenants, mon ancien chirurgien le docteur J... (P : 24).

$\rightarrow$ Mon ancien chirurgien, le docteur J., fait partie des intervenants.

\section{Tâche expérimentale}

\subsection{Objectifs et méthodes}

Pour approfondir cette étude, nous avons mené une tâche expérimentale dans le milieu universitaire des francophones natifs. Son but était de révéler :

i. si l'utilisation de la préposition entre était acceptable aux locuteurs natifs dans les phrases (4.1)(4.10), qui faisaient partie de notre corpus et dans lesquelles le nombre des constituants introduit par entre était souvent supérieur à l'indication numérique associée à cette préposition, c.-à-d. deux,

ii. s'ils allaient employer une autre préposition dans ces phrases,

iii. quelles représentations stéréotypées les prépositions entre et parmi véhiculaient auprès des locuteurs natifs,

iv. si et comment ils abrégeaient entre et parmi dans la communication médiée par téléphone (CMT) et par ordinateur (CMO).

Nous avons donc demandé aux sujets de remplir un questionnaire. D’abord, ils devaient compléter leurs données personnelles : l’origine géographique, lâge et la catégorie socio-professionnelle.

Ensuite, nous leur avons demandé si l'emploi de la préposition entre leur semblait acceptable ${ }^{23}$ dans les dix phrases proposées qui avaient été tirées du corpus (c.-à-d. Tableau 1, phrases 4.14.10). Il fallait choisir parmi ces trois réponses : « oui », « je ne sais pas» ou « non ». Les sujets pouvaient également proposer une « autre solution » (c.-à-d. des termes qui pourraient concurrencer ou remplacer la préposition entre). Lauthenticité des phrases francophones était une information connue des sujets, bien que la source de celles-ci ne leur eût pas été communiquée, c.-à-d. provenant de la littérature française contemporaine, afin d'éviter tout a priori positif de la part des sujets.

Enfin, nous avons posé aux sujets quatre questions supplémentaires sur la préposition entre et quatre autres sur la préposition parmi, à savoir :

i. ce qu'elles évoquaient pour eux,

ii. quels étaient leurs contraires,

iii. si et comment ils les abrégeaient dans les textos et dans les messages sur Internet et les réseaux sociaux. $^{24}$

23 Le mot " acceptable " utilisé dans la question signifie «possible en français pour les locuteurs natifs " : la question porte sur le jugement personnel de l'utilisation de cette préposition.

24 Nous avons inséré cette question dans le questionnaire car nous sommes également intéressés par les modifications orthographiques (simplifications) dans la CMT et CMO, dont létude se développe récemment. 


\subsection{Profil des sujets}

La présente tâche, qui visait des francophones natifs, s'est déroulée dans le milieu universitaire, en deux étapes : en décembre 2016 dans la ville de Lorient, en Bretagne, et en avril 2017 dans la ville de Clermont-Ferrand, en Auvergne. Ci-dessous suivent les informations principales sur le nombre, l'origine, l'âge et la catégorie socio-professionnelle des sujets.

155 sujets y ont participé au total. 152 personnes étaient originaires de France, 2 personnes venaient de Mayotte et 1 personne du Sénégal, mais ils étudiaient tous en France.

142 sujets, c.-à-d. une large majorité, étaient âgés de 18 à 30 ans. 2 sujets avaient moins de 18 ans, 9 sujets entre 31 et 50 ans et 2 personnes avaient plus de 50 ans.

Enfin, 151 sujets faisaient leurs études de licence ou de master, soit à la Faculté de Lettres, Langues, Sciences Humaines et Sociales de l'Université de Bretagne-Sud, soit à l'UFR de Lettres, Langues et Sciences Humaines de l'Université Clermont Auvergne. Parmi les 4 autres sujets, il y avait 2 maîtres de conférence, 1 manageur et 1 stylicien.

Ainsi, ce public était assez homogène, représenté par des étudiants d’origine française dans le groupe d'âge 18-30 ans.

\subsection{Résultats}

Dans cette sous-section, nous montrerons les résultats de la tâche expérimentale.

4.3.1 Consigne : «L'emploi de la préposition entre est-il pour vous acceptable dans les phrases suivantes? Veuillez indiquer « oui » / « je ne sais pas » ( ? ) / ou « non ». Vous pouvez aussi proposer une autre solution.

\begin{tabular}{|c|c|c|c|c|c|}
\hline $\mathbf{N}^{o}$ & Phrase & Oui & $?$ & Non & Autre solution \\
\hline 4.1 & $\begin{array}{l}\text { À Madrid, il n’est plus toléré de déambuler dans les rues entre des pan- } \\
\text { neaux d'affichage publicitaire, c'est-à-dire en " sandwich ». }\end{array}$ & 94 & 33 & 28 & $\begin{array}{l}\text { avec (6) } \\
\text { au milieu de (3) } \\
\text { parmi (1) }\end{array}$ \\
\hline 4.2 & $\begin{array}{l}\text { À peine débouchait-il sur la place qu'il était happé par la foule qui s’y } \\
\text { pressait ; il avait beau faire, entre les calèches, les grappes de touristes } \\
\text { et les vendeurs de babioles et de roses, il se frayait difficilement un } \\
\text { passage. }\end{array}$ & 122 & 16 & 17 & $\begin{array}{l}\text { parmi (8) } \\
\text { au milieu des (1) }\end{array}$ \\
\hline 4.3 & $\begin{array}{l}\text { Nous prenons une sorte de ruelle, entre des sortes de remparts, avec } \\
\text { une galerie qui la traverse à la hauteur d'un } 1^{\mathrm{er}} \text { étage. }\end{array}$ & 106 & 18 & 31 & $\begin{array}{l}\text { entourés de }(2) \\
\text { au milieu de }(2) \\
\text { au centre de (1) }\end{array}$ \\
\hline 4.4 & $\begin{array}{l}\text { Bataille avec les feuilles de fusain qui ornent la nappe entre Janine, } \\
\text { Lopez, Paulette et moi. }\end{array}$ & 133 & 11 & 11 & $\begin{array}{l}\operatorname{avec}(2) \\
\operatorname{parmi}(1)\end{array}$ \\
\hline 4.5 & $\begin{array}{l}\text { [...], mes vacances étant inégalement partagées entre le cycle, la pé- } \\
\text { tanque, la pêche, la lettre d'amour [...] et les tournées rurales d’apéritifs } \\
\text { à la terrasse de mon Q.G. }\end{array}$ & 137 & 10 & 8 & parmi (1) \\
\hline
\end{tabular}




\begin{tabular}{|c|l|c|c|c|c|}
\hline $\mathbf{N}^{\mathbf{0}}$ & Phrase & Oui & $?$ & Non & Autre solution \\
\hline 4.6 & $\begin{array}{l}\text { Nous organisions des « courses », entre copains, tout autour des écoles } \\
\text { Berthelot, à Villeneuve-Saint-Georges, [...] ! }\end{array}$ & 153 & 1 & 1 & avec (4) \\
\hline 4.7 & Peu d'entre eux sont arrivés à vélo. & 151 & 0 & 4 & parmi (1) \\
\hline 4.8 & $\begin{array}{l}\text { Ce disant, il le fait ruisseler entre ses doigts calleux, scintillant de mille } \\
\text { particules de gypse, de quartz et de mica, grains de sel, bris infimes de } \\
\text { coquillages. }\end{array}$ & 151 & 3 & 1 & dans (1) \\
\hline 4.9 & $\begin{array}{l}\text { En approchant, d'entre les mélèzes, j’ai vu un filet de fumée sortir du } \\
\text { toit, tout droit, tranquille; j’ai su qu'il était toujours là. }\end{array}$ & 63 & 48 & 44 & $\begin{array}{l}\text { parmi (7) } \\
\text { à travers (4) } \\
\text { au milieu de (3) } \\
\text { entre (2) } \\
\text { depuis (2) } \\
\text { du milieu de (1) } \\
\text { près des } \\
\text { mélèzes (1) }\end{array}$ \\
\hline 4.10 & $\begin{array}{l}\text { S'est-il retrouvé, au mauvais moment, au centre d’une affaire louche, } \\
\text { d'hôtel inconstructible, d'un conflit entre associés? }\end{array}$ & 146 & 5 & $\begin{array}{l}\text { d'un conflit } \\
\text { d'associés (2) }\end{array}$ \\
\hline
\end{tabular}

Tableau 1. Acceptabilité de la préposition entre

Il est remarquable quaucune phrase tirée du corpus littéraire et comportant la préposition entre n’ait été jugée acceptable par la totalité des sujets.

La phrase (4.9) a été rejetée par le plus grand nombre des sujets. Elle a également obtenu le plus grand nombre des réponses « je ne sais pas ». 7 sujets ont proposé la solution « parmi les mélèzes », 4 personnes « à travers les mélèzes » et 3 personnes « au milieu des mélèzes ». Parmi les autres reformulations suggérées figuraient celles-ci : «du milieu des mélèzes », « entre les mélèzes », « depuis les mélèzes » et " près des mélèzes ». Dans cette phrase, le locuteur s'approchait des mélèzes. Les prépositions d’entre, à travers et entre suggèrent une idée de passage. Celle-ci manque avec les expressions parmi (idée de différenciation du lieu traversé : ce ne sont pas les mêmes mélèzes, les mélèzes sont différenciés et indéfinis ; vision partielle à travers les mélèzes), au milieu de, du milieu de et près de.

Puis, la phrase (4.1) n'est acceptable que pour 94 répondants, les autres ne savent pas ou la jugent inacceptable. On y parle de «l'homme sandwich ». Les syntagmes " entre des panneaux d'affichage publicitaire ", " avec des panneaux d'affichage publicitaire » (proposée 6 fois et désignant la coprésence) et " au milieu des panneaux d’affichage publicitaire » (proposée 3 fois et soulignant une dimension spatiale) ont un sens similaire. Lemploi de la préposition parmi (proposée 1 fois) serait étrange en raison de l'image de l'homme sandwich qui porte deux panneaux d'affichage publicitaire.

Ensuite, dans la phrase (4.3), approuvée par 106 sujets sur 155, 5 sujets suggèrent ces expressions : "entourés de / au milieu de / au centre de sortes de remparts ». Elles ont toutes un sens similaire, au centre de étant plus précis qu'au milieu de.

En revanche, une large majorité de personnes a notamment approuvé les phrases (4.6), (4.7), (4.8), (4.10) et aussi, dans une moindre mesure, (4.4) et (4.5). 
C'est dans la phrase (4.2) que le plus grand nombre des sujets, précisément 8, ont proposé d'employer la préposition parmi : "parmi les calèches, les grappes de touristes et les vendeurs de babioles et de roses ». Les trois prépositions concurrentes, entre, parmi et au milieu de, ont un sens similaire, mais ne sont pas synonymiques : entre suggère une idée de passage entre trois ensembles homogènes (calèches ${ }^{\mathrm{E} 1}$ - touristes $^{\mathrm{E} 2}$ - vendeurs ${ }^{\mathrm{E} 3}$ ), tandis que parmi confond les trois ensembles et évoque une idée de passage au milieu d'un ensemble hétérogène, obtenu du mélange de trois sous-ensembles (calèches ${ }^{\mathrm{SE} 1}+$ touristes $^{\mathrm{SE} 2}+$ vendeurs $^{\mathrm{SE} 3}$ ) ; de plus, la personne avance en faisant des zigzagues.

Dans (4.4), entre et parmi sont plus passifs, avec est plus actif : «bataille ... entre / parmi / avec Janine, Lopez, Paulette et moi ». Le complément comitatif en avec peut être paraphrasé : " nous nous battons ensemble ». Rappelons néanmoins que la phrase originale pourrait être ambigüe (voir la sous-section 3.1).

L'emploi de la préposition parmi dans (4.5), suggéré par un seul sujet, est incorrect, car parmi risque de prêter à confusion les cinq termes.

La préposition avec dans (4.6), proposée par 4 sujets, exige un déterminant ou un nom propre : « entre copains » mais « avec nos/les copains ».

Dans (4.7), d'entre marque la séparation, la division d'un groupe de personnes en deux parts, mais parmi, qui également introduit un champ de sélection, a un sens plus étendu : "peu d’entre / parmi eux ».

Dans (4.8), entre distingue quelque chose, alors que dans, suggéré une seule fois, réunit : «il le fait ruisseler entre / dans ses doigts calleux ». Les doigts d'une personne représentent des parties qui sont intrinsèquement connectées et fonctionnellement dépendantes, c'est pourquoi, dans cette phrase, parmi ne peut pas se substituer à entre (ce qui n'est, soit dit en passant, proposé par aucun sujet).

Semblablement dans (4.10), entre distingue et de réunit ; entre précise davantage le rapport, de est plus abstrait et polyvalent : « un conflit entre associés / d'associés ».

Les réponses des sujets dans cette sous-section ont mis au jour les prépositions concurrentes qui peuvent être appelées par entre, selon la variabilité de son sens. Il s'agit notamment de parmi (19 fois), avec (12 fois) et au milieu de (9 fois). Le nombre des constituants introduit par entre n'a pas eu un impact considérable sur l'acceptabilité de cette préposition, sauf peut-être dans les phrases (4.2) et (4.9).

\subsubsection{Question : «Selon vous, quévoquent les prépositions entre et parmi?»}

\begin{tabular}{|c|l|c|}
\hline $\mathbf{N}^{\mathbf{0}}$ & Sens & Nb de réponses \\
\hline 1. & au milieu (de)* & 55 \\
\hline 2. & un espace, une position** & 26 \\
\hline 3. & un choix & 18 \\
\hline 4. & parmi ( ${ }^{*}$ parmis) & 13 \\
\hline $5 .-6$. & à l'intérieur (de), au centre (de) & 8 \\
\hline
\end{tabular}




\begin{tabular}{|c|l|c|}
\hline $\mathbf{N}^{\mathbf{o}}$ & Sens & Nb de réponses \\
\hline $5 .-6$. & un lien, une relation & 8 \\
\hline 7. & avec & 4 \\
\hline $8 .-9$. & entouré par plusieurs choses & 3 \\
\hline $8 .-9$. & un élément (isolé ou non) dans un ensemble & 3 \\
\hline 10. & une comparaison & 2 \\
\hline 11. & sans réponse & 18 \\
\hline
\end{tabular}

Tableau 2. Sens évoqués par la préposition entre

* Par ex. « quelque chose qui se trouve au milieu de deux choses », " être au milieu de deux choses minimum », " au milieu de plusieurs choses ». Une personne a présenté cette comparaison : «Entre évoque une disposition d'un objet ou d'un sujet par rapport à d'autres au milieu. Parmi évoque la disposition d'un objet ou d'un sujet dans un groupe. »

** Par ex. " position centrale, sans en faire partie».

\begin{tabular}{|c|l|c|}
\hline $\mathbf{N}^{\circ}$ & Sens & Nb de réponses \\
\hline 1. & un « ensemble » dont fait partie quelque chose ou quelqu'un* & 31 \\
\hline 2. & dans** & 26 \\
\hline 3. & au milieu de : une chose au milieu d'autres $* *$ & 24 \\
\hline $4 .-5$. & « avec » quelque chose & 16 \\
\hline $4 .-5$. & un choix & 16 \\
\hline $6 .-7$. & à l'intérieur de : à l'intérieur d'un groupe & 12 \\
\hline $6 .-7$. & entre & 12 \\
\hline 8. & «au sein de » : au sein d'un groupe & 4 \\
\hline 9. & sans réponse & 17 \\
\hline
\end{tabular}

Tableau 3. Sens évoqués par la préposition parmi

* Par ex. « contrairement à entre, fait partie du groupe ».

** Par ex. " un élément dans un ensemble ", " une chose dans un groupe », " quelque chose dans autre chose ».

*** Par ex. " un placement au milieu de plusieurs éléments », " elle permet de situer un élément au milieu d'autres ».

Dans les deux tableaux ci-dessus figurent les sens les plus fréquemment indiqués par nos sujets, chacun d’entre eux pouvait fournir une ou plusieurs réponses. Les sens rarement évoqués (c.-à-d. 1 ou 2 fois) n'y apparaissent pas.

Concernant entre, nous avons obtenu 137 réponses, 18 personnes, au contraire, n’ont pas réagi. 55 sujets, c'est-à-dire le plus grand nombre, associent entre à la locution au milieu (de), pourtant il s'agit du sens premier de parmi. 26 sujets le lient aux notions d'espace et de position et 18 sujets ont mentionné le concept de choix. Pour définir la préposition entre, 13 personnes se sont servis de la préposition parmi, souvent orthographiée incorrectement ${ }^{*}$ parmis (peut-être en raison de la ressemblance orthographique avec la préposition hormis). Personne n’a explicitement mentionné une valeur temporelle. 
Pour ce qui est de parmi, le nombre de réponses fournies sélève à 138, donc 17 personnes n’ont pas répondu. D’après les sujets, parmi évoque notamment ces idées : un ensemble dont fait partie quelque chose ou quelqu'un (31 fois), la préposition dans (26 fois), la locution au milieu de : une chose au milieu d'autres (24 fois), la préposition avec (16 fois) et la notion de choix (16 fois). Notons que de nombreux sens mentionnés par les sujets sont très proches et associés aux notions d’ensemble, de groupe, d'intériorité et d'appartenance à un tout. Ils confirment les définitions de Martinet et de Grevisse - Goosse présentées au début de la section 3.2. Les prépositions dans et au milieu de reflètent l’emploi spatial. Le sens premier de parmi, c.-à-d. au milieu de, noccupe que le $3^{\mathrm{e}}$ rang. En définissant la préposition parmi, 12 sujets ont eu recours à la préposition entre.

\subsubsection{Question : «Quels sont leurs contraires?»}

102 sujets ont répondu à la question portant sur le contraire possible de entre. Parmi la multiplicité de réponses fournies, ces termes ont été mentionnés le plus fréquemment : hors (de) et en dehors (de) (37 fois), à côté (de) (30 fois) et autour ${ }^{25}$ (18 fois). Il s'agit donc de termes marquant l'exclusion et la position dans l'espace (rappelons que entre, de même que parmi, exprime l'inclusion). Seuls 3 sujets ont écrit que entre n’a pas de contraire. Et 2 personnes ont proposé les prépositions devant et derrière, qui, dans ce cas, font référence au trinôme spatial devant - entre - derrière (voir Cervoni 1991 : 161).

95 sujets ont réagi à la question concernant le contraire possible de parmi. Ils ont proposé en particulier des termes marquant l'exclusion, comme hors (de) et en dehors (de) (57 fois), exclu (8 fois), hormis (6 fois) et à part (4 fois). Un sujet a répondu que parmi n’a aucun contraire.

\subsubsection{Question : «Abrégez-vous entre et parmi dans les textos et les messages sur Internet et les réseaux sociaux ? OUI / NON. Si OUI, comment?»}

Tous les sujets sauf un (qui n’a pas indiqué la forme utilisée) ont déclaré ne pas abréger entre et parmi dans les textos et les messages sur Internet et les réseaux sociaux.

\section{Remarques conclusives}

La variabilité de sens des prépositions entre et parmi dans notre corpus littéraire datant de 2013 est déterminée par la thématique des œuvres, d’une part, et, d’autre part, par la personnalité des auteurs, leur idiolecte et leur intention artistique. Du point de vue syntaxique, les deux prépositions ont essentiellement pour régime un syntagme nominal pluriel, même si parmi attire également les noms collectifs. Comme nous pouvons le voir dans notre corpus, l'indication numérique de entre ne se limite pas à deux éléments : cette préposition peut introduire également des entités multiples, de même que parmi. Les valeurs codésignées par entre sont d’ordre spatial, tempo- 
rel et notionnel. Parmi, qui a également des emplois spatiaux et notionnels, ne s'applique pas au temps. Entre et parmi sont commutables dans certains contextes, mais avec des nuances de sens. Lorsqu'elles expriment une relation spatiale, les deux prépositions génèrent une représentation différente en ce qui concerne l'occupation de l'espace. Celle de parmi paraît moins précise, sa cible est localisée quelque part dans le champ spatial du site, alors que celle de entre, définie plus nettement, est ordonnée conformément aux éléments constituant le site. Comme l'utilisation spatiale de parmi fait partie de notre corpus, datant de 2013, et comme elle est évoquée par nos sujets, elle ne devrait pas être considérée comme marginale ou vieillie, au même titre que parmi la campagne (voir la note $\mathrm{n}^{\circ} 2$ ).

Quelques observations intéressantes ont pu être faites grâce à notre tâche expérimentale qui a été effectuée dans le milieu universitaire français, parmi les locuteurs natifs, entre 2016 et 2017, et qui englobait 155 sujets. Premièrement, aucune phrase tirée du corpus littéraire et comportant la préposition entre n'a été jugée acceptable par la totalité des sujets. Il faut néanmoins tenir compte du fait que les textes littéraires, anciens ou actuels, peuvent contenir quelques particularités linguistiques, y compris au niveau prépositionnel, qui sont dues aux styles de leurs auteurs. C'est pourquoi certains emplois spécifiques de entre ou de parmi ne devraient pas être considérés comme des emplois typiques, ce dont témoignent également les réponses des sujets, qui ne sont pas toujours unanimes. De plus, les sujets auraient pu être influencés par l'ambiguïté (4.4) ou la complexité de certaines phrases (4.9). Deuxièmement, entre évoque notamment la locution au milieu (de) (rappelons qu'il s'agit du sens premier de parmi), ensuite les notions d'espace et de position, et après, le concept de choix. Parmi appelle essentiellement un ensemble dont fait partie quelque chose ou quelqu'un, puis la préposition dans, et après, la locution au milieu de (une chose au milieu d'autres). Troisièmement, concernant leurs contraires respectifs, les sujets ont essentiellement mentionné les termes marquant l'exclusion : hors (de) et en dehors (de). Même si entre et parmi ne forment pas un couple antonymique prototypique avec une autre préposition, comme p. ex. sur ou dans (c.-à-d. sur/sous, dans/hors de), ils aident à exprimer l'inclusion. Quatrièmement, les sujets n'abrègent pas ces deux prépositions dans la communication médiée par téléphone et par ordinateur, ce qui pourrait suggérer une nouvelle étude sur l'abréviation des prépositions variées. Enfin, lors de l'étude, plusieurs sujets ont orthographié de manière incorrecte la préposition parmi. Nous émettons l'hypothèse que la source de l'orthographe incorrecte parmis trouve son origine en raison de la ressemblance avec la préposition hormis.

\section{Références bibliographiques}

Cadiot, P. (1997). Les Prépositions abstraites en français. Paris : Armand Colin / Masson.

Cervoni, J. (1991). La préposition, étude sémantique et pragmatique. Paris/Louvain-la-Neuve : Duculot.

Franckel, J.-J. ; \& Paillard, D. (2007). Grammaire des prépositions. Paris : Éditions Ophrys.

Gréa, P. (2015). Entre et parmi : deux perspectives sur la pluralité. Travaux de linguistique, 70 (1), 7-38.

Grevisse, M. ; \& Goosse, A. (2008). Le Bon usage. 14éd. Bruxelles : De Boeck \& Larcier. 
Hilgert, E. (2011). Parmi / entre / d'entre les $N$ et le problème de leur interprétation spatiale. In L'expression de l'espace et du temps en français : quelles formes pour quels sens ? Colloque de Belgrade, 23-26 mars $2011<$ http://docplayer.fr/27449667-Parmi-entre-d-entre-les-n-et-le-probleme-de-leur-interpretationspatiale.html> . (2012). Les prépositions ensemblistes et la question de leur emploi spatial. In B. Fagard, \& D. Stojic (Éds.), Corela - Langue, espace, cognition. <http://corela.revues.org/2808>

Kwon-Pak, S. N. (2006). Entre vs. Parmi : deux prépositions au centre de la partition. In G. Kleiber, A. Schnedecker, \& A. Theissen (Éds.), La Relation partie-tout (pp. 651-668). Leuven : Peeters.

Martinet, A. (dir.) (1979). Grammaire fonctionnelle du français. Paris : Didier.

Rey, A. (dir.) (1992 \& 2010). Dictionnaire historique de la langue française. Paris : Dictionnaires Le Robert.

Šabršula, J. (1989). Les espèces de relation - Nové kapitoly z rozboru moderní francouzštiny IV. Praha : Univerzita Karlova v Praze / SPN.

Vaguer, C. (2008). Classement syntaxique des prépositions simples du français. In D. Leeman (Éd.), Langue française $n^{\circ} 157$ : Énigmatiques prépositions (pp. 20-36). Paris : Larousse.

\section{Sitographie}

Frantext [en ligne]. [2017-IV-18]. <http://www.frantext.fr/>

Le Parisien, Nîme : intoxication massive parmi le personnel de l'hôpital. <http://www.leparisien.fr/languedoc-roussillon/nimes-intoxication-massive-parmi-le-personnel-de-l-hopital-12-08-2016-6035701. php> 